\title{
Born rule in quantum and classical mechanics
}

\author{
Paul Brumer and Jiangbin Gong \\ Chemical Physics Theory Group and Center for Quantum Information and Quantum Control, University of Toronto, \\ Toronto, Ontario, Canada M5S 3 H6
}

(Received 17 January 2006; published 19 May 2006)

\begin{abstract}
Considerable effort has been devoted to deriving the Born rule [i.e., that $|\psi(x)|^{2} d x$ is the probability of finding a system, described by $\psi$, between $x$ and $x+d x]$ in quantum mechanics. Here we show that the Born rule is not solely quantum mechanical; rather, it arises naturally in the Hilbert-space formulation of classical mechanics as well. These results provide insights into the nature of the Born rule, and impact on its understanding in the framework of quantum mechanics.
\end{abstract}

DOI: 10.1103/PhysRevA.73.052109

PACS number(s): 03.65.Sq, 03.65.Ca

\section{INTRODUCTION}

The Born rule [1] postulates a connection between deterministic quantum mechanics in a Hilbert-space formalism with probabilistic predictions of measurement outcomes. It is typically stated [2] as follows (without considering degeneracies): if an observable $\hat{O}$, with eigenstates $\left\{\left|O_{i}\right\rangle\right\}$ and spectrum $\left\{O_{i}\right\}$, is measured on a system described by the state vector $|\psi\rangle$, the probability for the measurement to yield the value $O_{i}$ is given by $\left|\left\langle O_{i} \mid \psi\right\rangle\right|^{2}$. Alternatively, in the density matrix formulation used below, this rule states that the probability is $\operatorname{Tr}\left[\rho_{\psi} \rho_{O_{i}}\right]$, where $\rho_{\psi}=|\psi\rangle\langle\psi|$ and $\rho_{O_{i}}=\left|O_{i}\right\rangle\left\langle O_{i}\right|$. Most familiar is the textbook example that the probability of observing a system that is in a state $\psi$ in the coordinate range $x$ to $x+d x$ is given by $|\langle x \mid \psi\rangle|^{2} d x$. Born's rule appears as a fundamental postulate in quantum mechanics and is thus far in agreement with experiment. Hence, there is intense interest in providing an underlying motivation for, or derivation of, this rule.

Gleason's theorem [3], for example, provides a formal motivation of the Born rule, but it is a purely mathematical result about vectors in Hilbert spaces and does not provide insight into the physics of this postulate. For this reason there have been several attempts to provide a physical derivation of the Born rule. For example, Deutsch showed the possibility of deriving the Born rule from "the nonprobabilistic axioms of quantum theory" and "the nonprobabilistic part of classical decision theory" [4]. Deutsch's approach was criticized by Barnum et al. [5] but was recently reinforced by Saunders [6]. Hanson [7] and Wallace [8] analyzed the connection between possible derivations of the Born rule and Everett's many-worlds interpretation of quantum mechanics. Zurek recently proposed a significantly new approach, the so-called envariance approach [9], for deriving the Born rule from within quantum mechanics. This approach, totally different from Deutsch's method, was recently analyzed in detail by Schlosshauer and Fine [2] and by Zurek [10]. Zurek's envariance approach has also been analyzed and modified by Barnum [11]. All these studies have attracted considerable interest in deriving Born's rule by making some basic assumptions about quantum probabilities or expectation values of observables.

The Born rule is not expected to violate any future experiments. In this sense, even a strict derivation of the Born rule will not help predict any experimentally new physics. However, understanding the origin of the Born rule is important for isolating this postulate from other concepts in quantum mechanics and for understanding what is truly unique in quantum mechanics as compared with the classical physics. For example, based on the above-mentioned efforts to derive the Born rule [2,4,6,9-11], it seems now clear that the physical origin of Born's rule is unrelated to the details (e.g., wave function collapse) of quantum measurement processes.

The main purpose of this work is to show that Born's rule is not solely quantum mechanical and that it arises naturally in the Hilbert space formulation of classical mechanics. As such, the Born rule connecting probabilities with eigenvalues and eigenfunctions is not as "quantum" as it sounds. Indeed, quantum-classical correspondence, which played no role in Born's original considerations [1,12], can then be arguably regarded as an interesting motivation for the Born rule in quantum mechanics. Similarly, exposing the Born rule in classical mechanics should stimulate new routes to understanding the physical origin of this rule in quantum mechanics. In particular, new and interesting questions can be asked in connection with the previous derivations of the Born rule.

To demonstrate that the Born rule exists in both quantum and classical mechanics we (1) recall that both quantum and classical mechanics can be formulated in the Hilbert space of density operators [13-18], that the quantum and classical systems are represented by vectors $\rho$ and $\rho_{c}$, respectively, in that Hilbert space, and that $\rho$ and $\rho_{c}$ can be expanded in eigenstates of a set of commuting quantum and classical superoperators, respectively; (2) show that the quantummechanical Born rule can be expressed in terms of the expansion coefficient of a given density associated with eigendistributions of a set of superoperators in the Hilbert space of density matrix; and (3) show that the classical interpretation of the phase-space representation of $\rho_{c}$ as a probability density allows the extraction of Born's rule in classical mechanics, and gives exactly the same structure as the quantum-mechanical Born rule.

These results suggest that the quantum-mechanical Born rule not only applies to cases of large quantum numbers, but also has a well-defined purely classical limit. Hence, independent of other subtle elements of the quantum theory, the inherent consistency with the classical Born rule for the macroscopic world imposes an important condition on any 
eigenvalue-eigenfunction-based probability rule in quantum mechanics.

\section{THE QUANTUM-MECHANICAL BORN RULE IN DENSITY MATRIX FORMALISM}

Consider first quantum mechanics in the Hilbert space of the density matrix $[16,17]$. Given an operator $\hat{O} \equiv \hat{K}_{N}$ for a system of $N$ degrees of freedom, we first consider the (classically) integrable case where there exist $N$ independent and commuting observables $\hat{K}_{i}, i=1, \ldots, N$. Another extreme, the chaotic case, will be discussed in Sec. IV. For convenience we also assume that the $\hat{K}_{i}, i=1,2, \ldots, N$, have a discrete spectrum, but the central result below applies to cases with a continuous spectrum as well. The complete set of commuting superoperators in the quantum Hilbert space can be constructed as

$$
\frac{1}{\hbar}\left[\hat{K}_{i},\right], \quad \frac{1}{2}\left[\hat{K}_{i},\right]_{+} \quad(i=1,2, \ldots, N),
$$

where $[$,$] denotes the commutator and [,]_{+}$denotes the anticommutator, i.e., $[\hat{A}, \hat{B}]=\hat{A} \hat{B}-\hat{B} \hat{A},[\hat{A}, \hat{B}]_{+}=\hat{A} \hat{B}+\hat{B} \hat{A}$. The simultaneous eigendensities of the complete set of superoperators are denoted $\rho_{\alpha, \beta}$. That is,

$$
\begin{gathered}
\frac{1}{2}\left[\hat{K}_{i}, \rho_{\alpha, \beta}\right]_{+}=\alpha_{i} \rho_{\alpha, \beta}, \\
\frac{1}{\hbar}\left[\hat{K}_{i}, \rho_{\alpha, \beta}\right]=\beta_{i} \rho_{\alpha, \beta},
\end{gathered}
$$

where $\alpha \equiv\left(\alpha_{1}, \alpha_{2}, \ldots, \alpha_{N}\right)$ is the collection of eigenvalues associated with $\frac{1}{2}\left[\hat{K}_{i},\right]_{+}$, and $\beta \equiv\left(\beta_{1}, \beta_{2}, \ldots, \beta_{N}\right)$ is the collection of eigenvalues associated with $\frac{1}{\hbar}\left[\hat{K}_{i},\right]$.

The state of the quantum system is described by an arbitrary density matrix $\rho$ in the Hilbert space under consideration, and can be expanded in terms of the basis states $\rho_{\alpha, \beta}$ as

$$
\rho=\sum_{\alpha, \beta} D_{\alpha, \beta} \rho_{\alpha, \beta},
$$

where the sum is over all eigendensities. The sum should be understood as an integral if the spectrum is continuous. Clearly, the expansion coefficients in Eq. (3) are given by

$$
D_{\alpha, \beta}=\operatorname{Tr}\left[\rho \rho_{\alpha, \beta}^{\dagger}\right] .
$$

Equations (3) and (4), i.e., the expansion of $\rho$ in terms of the eigendensities $\rho_{\alpha, \beta}$, are central to the analysis later below.

Consider now the quantum probability $\mathcal{P}_{Q}\left(\mathbf{K}^{\prime}\right)$ of finding the quantum observables $\hat{K}_{i}$ with eigenvalues $K_{i}^{\prime}, i$ $=1,2, \ldots, N$, given that the system is in state $\rho$. We show here that the Born rule is then equivalent to the statement that $\mathcal{P}_{Q}\left(\mathbf{K}^{\prime}\right)$ must be proportional to the expansion coefficient $D_{\mathbf{K}^{\prime}, \boldsymbol{0}}$ of the given density $\rho$ associated with the common eigendistribution $\rho_{\mathbf{K}^{\prime}, \mathbf{0}}$ of superoperators $\frac{1}{2}\left[\hat{K}_{i},\right]_{+}$with eigenvalues $K_{i}^{\prime}$ and of superoperators $\frac{1}{\hbar}\left[\hat{K}_{i}\right.$, ] with eigenvalue zero. To see this, consider first a quantum density for a pure quantum state, e.g., $\rho=|\psi\rangle\langle\psi|$ (the extension to mixed states is straightforward). Then the Born rule gives that

$$
\mathcal{P}_{Q}\left(\mathbf{K}^{\prime}\right)=\left|\left\langle\psi \mid \mathbf{K}^{\prime}\right\rangle\right|^{2}=\operatorname{Tr}\left[\left|\mathbf{K}^{\prime}\right\rangle\left\langle\mathbf{K}^{\prime} \| \psi\right\rangle\langle\psi|\right]=\operatorname{Tr}\left[\left|\mathbf{K}^{\prime}\right\rangle\left\langle\mathbf{K}^{\prime}\right| \rho\right],
$$

where $\left|\mathbf{K}^{\prime}\right\rangle$ is a common and normalized eigenfunction of operators $\hat{K}_{i}, i=1,2, \ldots, N$. However,

$$
\frac{1}{2}\left[\hat{K}_{i},\left|\mathbf{K}^{\prime}\right\rangle\left\langle\mathbf{K}^{\prime}\right|\right]_{+}=K_{i}^{\prime}\left|\mathbf{K}^{\prime}\right\rangle\left\langle\mathbf{K}^{\prime}\right| ; \quad\left[\hat{K}_{i},\left|\mathbf{K}^{\prime}\right\rangle\left\langle\mathbf{K}^{\prime}\right|\right]=0,
$$

so that $\left|\mathbf{K}^{\prime}\right\rangle\left\langle\mathbf{K}^{\prime}\right|$ is seen to be the common eigendistribution of superoperators $\frac{1}{2}\left[\hat{K}_{i},\right]_{+}$with eigenvalues $K_{i}^{\prime}$ and of superoperators $\frac{1}{\hbar}\left[\hat{K}_{i},\right]$ with eigenvalue zero. That is,

$$
\left|\mathbf{K}^{\prime}\right\rangle\left\langle\mathbf{K}^{\prime}\right|=\rho_{\mathbf{K}^{\prime}, \mathbf{0}} .
$$

Equations (4), (5), and (7), then lead to

$$
\mathcal{P}_{Q}\left(\mathbf{K}^{\prime}\right)=D_{\mathbf{K}^{\prime}, \mathbf{0}} .
$$

Equation (8) is a general restatement of the quantummechanical Born rule based on the Hilbert-space structure of the density matrix.

Note that the multidimensional result of Eq. (5) has carefully accounted for possible degeneracies associated with $K_{N}^{\prime}$. That is, the total probability of observing $K_{N}^{\prime}$ would be obtained by summing $\mathcal{P}_{Q}\left(\mathbf{K}^{\prime}\right)$ with all possible $K_{i}^{\prime}, i$ $=1,2, \ldots,(N-1)$, a necessary procedure not explicitly stated in Born's rule.

\section{THE BORN RULE IN CLASSICAL MECHANICS}

Consider now classical mechanics. The mechanics has numerous equivalent formulations, such as Newton's laws, the Lagrangian and Hamiltonian formulations, Hamilton-Jacobi theory, etc. The less familiar Hilbert-space formulation of classical mechanics used below was first established by Koopman [13] and subsequently appreciated by, for example, Prigogine [14], Zwanzig [15], and us [17,18] in some theoretical considerations. This being the case, the above eigenvalue-eigenfunction structure is not unique to quantum mechanics, a fact that may not be well appreciated and that is exploited below.

It is convenient to introduce the classical picture in the phase-space representation, although abstract Hilbert-space formulations may be used as well. Consider then the same case as above. Let the classical limit of the Wigner-Weyl representation of $\hat{K}_{i}$ be $K_{i}(\mathbf{p}, \mathbf{q})$, where $(\mathbf{p}, \mathbf{q})$ are momentum- and coordinate-space variables. The complete set of commuting superoperators on the classical Hilbert space is then [17]

$$
i\left\{K_{j}(\mathbf{p}, \mathbf{q}),\right\}, \quad K_{j}(\mathbf{p}, \mathbf{q}), \quad j=1,2, \ldots, N,
$$

where $K_{j}(\mathbf{p}, \mathbf{q})$ are multiplicative operators and $\{$,$\} denotes$ the classical Poisson bracket. The simultaneous eigendensities of this complete set of classical operators, denoted $\rho_{\alpha^{c}, \beta^{c}}^{c}(\mathbf{p}, \mathbf{q})$, satisfy

$$
K_{j}(\mathbf{p}, \mathbf{q}) \rho_{\alpha^{c}, \beta^{c}}^{c}(\mathbf{p}, \mathbf{q})=\alpha_{j}^{c} \rho_{\alpha^{c}, \beta^{c}}^{c}(\mathbf{p}, \mathbf{q}),
$$




$$
i\left\{K_{j}(\mathbf{p}, \mathbf{q}), \rho_{\alpha^{c}, \beta^{c}}^{c}(\mathbf{p}, \mathbf{q})\right\}=\beta_{j}^{c} \rho_{\alpha^{c}, \beta^{c}}^{c}(\mathbf{p}, \mathbf{q}),
$$

where the notation $\alpha^{c}, \beta^{c}, \alpha_{j}^{c}$, and $\beta_{j}^{c}$ is introduced in parallel with the quantum case. An arbitrary classical probability density $\rho_{c}(\mathbf{p}, \mathbf{q})$ can be expanded as

$$
\rho_{c}(\mathbf{p}, \mathbf{q})=\sum_{\alpha^{c}, \beta^{c}} D_{\alpha^{c}, \beta^{c}}^{c} \rho_{\alpha^{c}, \beta^{c}}^{c}(\mathbf{p}, \mathbf{q}),
$$

where

$$
D_{\alpha^{c}, \beta^{c}}^{c}=\int d \mathbf{p} d \mathbf{q} \rho_{c}(\mathbf{p}, \mathbf{q})\left[\rho_{\alpha^{c}, \beta^{c}}^{c^{*}}(\mathbf{p}, \mathbf{q})\right] \equiv \operatorname{Tr}\left[\rho_{c} \rho_{\alpha^{c}, \beta^{c}}^{c \dagger}\right],
$$

and where the sum in Eq. (11) is over all eigendensities.

Consider now, within this formalism, the probability of finding $\mathbf{K}$ [with $\left.\mathbf{K} \equiv\left(K_{1}, K_{2}, \ldots, K_{N}\right)\right]$ between $\mathbf{K}^{\prime}$ and $\mathbf{K}^{\prime}$ $+d \mathbf{K}^{\prime}$. To proceed we make a canonical transformation between representations $(\mathbf{p}, \mathbf{q})$ and $(\mathbf{K}, \mathbf{Q})$, where $\mathbf{K}$ are the new momentum variables, and $\mathbf{Q} \equiv\left(Q_{1}, Q_{2}, \ldots, Q_{N}\right)$ denotes the new position variables conjugate to $\mathbf{K}$. The $\mathbf{Q}$ can be obtained by regarding $\mathbf{p}$ as a function of $\mathbf{q}$ and $\mathbf{K}$, defining the generating function $S(\mathbf{q}, \mathbf{K})=\int_{\mathbf{q}_{0}}^{\mathbf{q}} \mathbf{p}\left(\mathbf{q}^{\prime}, \mathbf{K}\right) \cdot d \mathbf{q}^{\prime}$, and then noting that $Q_{i}=\partial S / \partial K_{i}$ [19]. In this representation the classical eigendensities $\rho_{\alpha^{c}, \beta^{c}}^{c}$ take a rather simple form,

$$
\rho_{\alpha^{c}, \beta^{c}}^{c}(\mathbf{p}, \mathbf{q}) \Rightarrow \mathcal{R}_{\mathbf{K}^{\prime}, \mathbf{\Lambda}}(\mathbf{K}, \mathbf{Q})=\frac{1}{(2 \pi)^{N / 2}} \delta\left(\mathbf{K}^{\prime}-\mathbf{K}\right) \exp (i \boldsymbol{\Lambda} \cdot \mathbf{Q})
$$

with eigenvalues [20] $\alpha_{i}^{c}=K_{i}$ and $\beta_{i}^{c}=\Lambda_{i}$, for $i=1,2, \ldots, N$. The set of eigendistributions $\mathcal{R}_{\mathbf{K}^{\prime}, \Lambda}$ are complete and orthogonal, i.e.,

$$
\begin{aligned}
& \int d \mathbf{K} d \mathbf{\Lambda} \mathcal{R}_{\mathbf{K}, \mathbf{\Lambda}}^{*}\left(\mathbf{K}^{\prime}, \mathbf{Q}^{\prime}\right) \mathcal{R}_{\mathbf{K}, \mathbf{\Lambda}}\left(\mathbf{K}^{\prime \prime}, \mathbf{Q}^{\prime \prime}\right) \\
& =\delta\left(\mathbf{K}^{\prime}-\mathbf{K}^{\prime \prime}\right) \delta\left(\mathbf{Q}^{\prime}-\mathbf{Q}^{\prime \prime}\right), \\
& \int d \mathbf{K} d \mathbf{Q} \mathcal{R}_{\mathbf{K}^{\prime}, \mathbf{\Lambda}^{\prime}}^{*}(\mathbf{K}, \mathbf{Q}) \mathcal{R}_{\mathbf{K}^{\prime \prime}, \mathbf{\Lambda}^{\prime \prime}}(\mathbf{K}, \mathbf{Q}) \\
& =\delta\left(\boldsymbol{\Lambda}^{\prime}-\mathbf{\Lambda}^{\prime \prime}\right) \delta\left(\mathbf{K}^{\prime}-\mathbf{K}^{\prime \prime}\right) .
\end{aligned}
$$

The $\mathcal{R}_{\mathbf{K}^{\prime}, \Lambda}$ are "improper states," insofar as they contain $\delta$ functions. However, this is consistent with the fact that they are eigendistributions of classical superoperators that have continuous spectra. If desired, a rigged Hilbert space [21] can be used to include these states more formally.

Given a classical probability density $\rho_{c}(\mathbf{p}, \mathbf{q})$ that describes the state of the system, we can convert to the $\mathbf{K}, \mathbf{Q}$ representation to obtain $\rho_{c}^{\prime}(\mathbf{K}, \mathbf{Q}) \equiv \rho_{c}[\mathbf{p}(\mathbf{K}, \mathbf{Q}), \mathbf{q}(\mathbf{K}, \mathbf{Q})]$. The probability $\mathcal{P}_{c}\left(\mathbf{K}^{\prime}\right)$ of finding the observables between $\mathbf{K}^{\prime}$ and $\mathbf{K}^{\prime}+d \mathbf{K}$ is evidently given by

$$
\mathcal{P}_{c}\left(\mathbf{K}^{\prime}\right)=\left[\int d \mathbf{K} d \mathbf{Q} \delta\left(\mathbf{K}^{\prime}-\mathbf{K}\right) \rho_{c}^{\prime}(\mathbf{K}, \mathbf{Q})\right] d \mathbf{K} .
$$

This result has an enlightening interpretation in the Hilbert space formulation of classical mechanics, as can be seen by independently obtaining Eq. (15) using this approach. To do so, we first expand the given density in terms of the basis states $\mathcal{R}_{\mathbf{K}^{\prime}, \boldsymbol{\Lambda}}$. That is,

$$
\rho_{c}^{\prime}(\mathbf{K}, \mathbf{Q})=\int d \mathbf{K}^{\prime} d \mathbf{\Lambda} D_{\mathbf{K}^{\prime}, \boldsymbol{\Lambda}}^{c} \mathcal{R}_{\mathbf{K}^{\prime}, \mathbf{\Lambda}}(\mathbf{K}, \mathbf{Q}),
$$

where the expansion coefficients are given by the overlap integrals

$$
D_{\mathbf{K}^{\prime}, \mathbf{\Lambda}}^{c}=\int d \mathbf{K} d \mathbf{Q} \rho_{c}^{\prime}(\mathbf{K}, \mathbf{Q}) \mathcal{R}_{\mathbf{K}^{\prime}, \mathbf{\Lambda}}^{*}(\mathbf{K}, \mathbf{Q}) .
$$

Because classical probabilities in $\mathbf{K}$ that do not refer to $\mathbf{Q}$ are obtained by integrating over $\mathbf{Q}$, we obtain

$$
\mathcal{P}_{c}\left(\mathbf{K}^{\prime}\right)=\left[\int d \mathbf{Q} \rho_{c}^{\prime}\left(\mathbf{K}^{\prime}, \mathbf{Q}\right)\right] d \mathbf{K} .
$$

Substituting Eq. (16) into Eq. (18) and using Eq. (13) then yields

$$
\mathcal{P}_{c}\left(\mathbf{K}^{\prime}\right)=(2 \pi)^{N / 2} D_{\mathbf{K}^{\prime}, \mathbf{0}}^{c} d \mathbf{K}
$$

This result is equivalent to Eq. (15), but contains an important message from the perspective of mechanics in Hilbert space. That is, Eq. (19) indicates that, given a classical density $\rho_{c}^{\prime}$ that describes the state, the probability of finding the observable $K_{j}(j=1,2, \ldots, N)$ in a regime $\left(\mathbf{K}^{\prime}, \mathbf{K}^{\prime}+d \mathbf{K}\right)$, is proportional to the overlap between the given density $\rho_{c}^{\prime}$ and the common eigendistribution $\mathcal{R}_{\mathbf{K}^{\prime}, \mathbf{0}}$ of multiplicative operators $K_{j}$ with eigenvalues $K_{j}^{\prime}$ and of operators $i\left\{K_{j}\right.$, $\}$ with eigenvalue zero. This overlap is $D_{\mathbf{K}^{\prime}, 0}^{c}$, the expansion coefficient of $\rho_{c}^{\prime}$ in terms of $\mathcal{R}_{\mathbf{K}^{\prime}, \mathbf{0}}$. Significantly, this connection between classical probabilities and the overlap between the given classical-state density and a particular set of classical eigendistributions [Eq. (19)] is the direct analog of the quantum-mechanical Born rule in the density matrix formalism [Eq. (8)]. That is, Eq. (19) is the Born rule in classical mechanics.

\section{QUANTUM VERSUS CLASSICAL BORN RULE IN CHAOTIC CASES}

Classical-quantum correspondence of Hilbert-space structures in chaotic cases is more subtle and complicated than in integrable cases [17]. Nevertheless, results above indicate that only a particular set of eigendensities are relevant in understanding the quantum versus classical Born rule. Indeed, it is straightforward to show that our previous considerations also apply to cases where there do not exist $(N-1)$ observables that commute with $K_{N}(\mathbf{p}, \mathbf{q})$. For example, consider a chaotic spectrum case where $K_{N}(\mathbf{p}, \mathbf{q})$ does not commute with any other smooth phase-space functions $Z(\mathbf{p}, \mathbf{q})$, i.e., $\left\{K_{N}(\mathbf{p}, \mathbf{q}), Z(\mathbf{p}, \mathbf{q})\right\} \neq 0$ always holds. Following Ref. [17] we consider a function $\tau(\mathbf{p}, \mathbf{q})$ that satisfies $\left\{\tau(\mathbf{p}, \mathbf{q}), K_{N}(\mathbf{p}, \mathbf{q})\right\}=1$. Then the classical eigenfunction of the multiplicative operator $K_{N}(\mathbf{p}, \mathbf{q})$ and of the operator $i\left\{K_{N}(\mathbf{p}, \mathbf{q}),\right\}$ with eigenvalue $\lambda$ is given by $\xi \delta\left[K_{N}^{\prime}\right.$ $\left.-K_{N}(\mathbf{p}, \mathbf{q})\right] \exp [i \lambda \tau(\mathbf{p}, \mathbf{q})]$, where $\xi$ is a normalization con- 
stant. In particular, the eigenfunction with zero eigenvalue for the operator $i\left\{K_{N}(\mathbf{p}, \mathbf{q}),\right\}$ is $\xi \delta\left[K_{N}^{\prime}-K_{N}(\mathbf{p}, \mathbf{q})\right]$. This eigenfunction defines a $(2 N-1)$-dimensional hypersurface in phase space (whereas the eigenfunction $\mathcal{R}_{\mathbf{K}^{\prime}, \mathbf{0}}$ in the integral case defines an $N$-dimensional manifold). The overlap between this particular eigenfunction $\xi \delta\left[K_{N}^{\prime}-K_{N}(\mathbf{p}, \mathbf{q})\right]$ and a given phase-space probability density $\rho_{c}(\mathbf{p}, \mathbf{q})$, i.e.,

$$
P_{c}\left(K_{N}^{\prime}\right) \equiv \xi \int d \mathbf{p} d \mathbf{q} \rho_{c}(\mathbf{p}, \mathbf{q}) \delta\left[K_{N}^{\prime}-K_{N}(\mathbf{p}, \mathbf{q})\right],
$$

yields the probability $P_{c}\left(K_{N}^{\prime}\right) d K_{N}$ of finding $K_{N}(\mathbf{p}, \mathbf{q})$ lying in the regime $\left[K_{N}^{\prime}, K_{N}^{\prime}+d K_{N}\right]$. This is again in complete analogy to how the quantum probability $\mathcal{P}_{Q}\left(K_{N}^{\prime}\right)$ of finding the eigenvalue $K_{N}^{\prime}$ is determined, i.e., $\mathcal{P}_{Q}\left(K_{N}^{\prime}\right)$ is given by the overlap between a given quantum density $|\psi\rangle\langle\psi|$ and the eigenfunction of superoperator $\frac{1}{2}\left[\hat{K}_{N},\right]_{+}$with eigenvalue $K_{N}^{\prime}$ and of superoperator $\frac{1}{\hbar}\left[\hat{K}_{N},\right]$ with eigenvalue zero. Such a quantum eigenfunction is simply given by $\left|K_{N}^{\prime}\right\rangle\left\langle K_{N}^{\prime}\right|$, where $\left|K_{N}^{\prime}\right\rangle$ is the eigenfunction of $\hat{K}_{N}$ with eigenvalue $K_{N}^{\prime}$. These analyses make it clear that even for chaotic cases, the Born rule formulated in terms of eigendensities in the associated (classical or quantum) Hilbert space exists in both quantum and classical mechanics.

\section{CONCLUDING REMARKS}

In summary, with the quantum-mechanical Born rule formulated in the Hilbert space of density matrix, we have demonstrated an analogous Born rule in classical mechanics. In so doing we never assumed that the quantum density goes smoothly, in the classical limit, to a classical density that already has a clear probabilistic interpretation. Rather, we have simply assumed that a system in either quantum or classical mechanics is described by a density operator in Hilbert space, and that these density operators serve the same descriptive purpose in both mechanics. This, plus the decomposition of the operator in terms of eigendistributions of a set of commuting superoperators, sufficees to show that the Born rule applies in both quantum and classical mechanics. Hence, the quantum-mechanical Born rule appears to be very natural in the light of quantum-classical correspondence in how Hilbert-space structures embody measured probabilities. The recognition that Born's rule is not really a unique quantum element should complement, as well as impact upon, previous attempts to derive the Born rule [2,4,6,9-11]. Further, it motivates numerous questions, such as, can the quantum-mechanical Born rule be derived with fewer assumptions by taking advantage of the classical Born rule as a limit? how can one reconcile derivations involving purely quantum language with the existence of a classical Born rule?, etc. These, and related issues, are the subject of future work.

\section{ACKNOWLEDGMENTS}

This work was supported by a grant from the National Science and Engineering Research Council of Canada. We thank Professor. R. Kapral and Professor S. Whittington, University of Toronto, for comments on an earlier version of this manuscript.
[1] M. Born, Z. Phys. 37, 863 (1926).

[2] M. Schlosshauer and A. Fine, Found. Phys. 35, 197 (2005).

[3] A. M. Gleason, J. Math. Mech. 6, 885 (1957).

[4] D. Deutsch, Proc. R. Soc. London, Ser. A 455, 3129 (1999).

[5] H. Barnum, C. M. Caves, J. Finkelstein, C. A. Fuchs, and R. Schack, Proc. R. Soc. London, Ser. A 456, 1175 (2000).

[6] S. Saunders, Proc. R. Soc. London, Ser. A 460, 1771 (2004).

[7] R. Hanson, Fortschr. Phys. 33, 1129 (2003).

[8] D. Wallace, Stud. Hist. Philos. Mod. Phys. 34B, 415 (2003).

[9] W. H. Zurek Phys. Rev. Lett. 90, 120404 (2003).

[10] W. H. Zurek Phys. Rev. A 71, 052105 (2005).

[11] H. Barnum, e-print quant-ph/0312150.

[12] M. Born (unpublished).

[13] B. O. Koopman, Proc. Natl. Acad. Sci. U.S.A. 17, 315 (1931).

[14] I. Progogine, Non-Equilibrium Statistical Mechanics (Wiley,
New York, 1962).

[15] R. S. Zwanzig, Lectures in Theoretical Physics (Wiley, New York, 1961), Vol. 3, pp. 135.

[16] U. Fano, Rev. Mod. Phys. 29, 74 (1959).

[17] J. Wilkie and P. Brumer Phys. Rev. A 55, 27 (1997); 55, 43 (1997).

[18] C. Jaffé and P. Brumer, J. Phys. Chem. 88, 4829 (1984); J. Chem. Phys. 82, 2330 (1985).

[19] S. N. Rasband, Dynamics (Wiley, New York, 1983).

[20] The eigenvalues $\boldsymbol{\Lambda}$ can be either continuous or discrete, depending on the system. For example, the spectrum comprises integers when, for an integrable system, the $\mathbf{K}, \mathbf{Q}$ are actionangle variables.

[21] A. Bohm, Quantum Mechanics: Foundations and Applications (Springer, New York, 1993). 